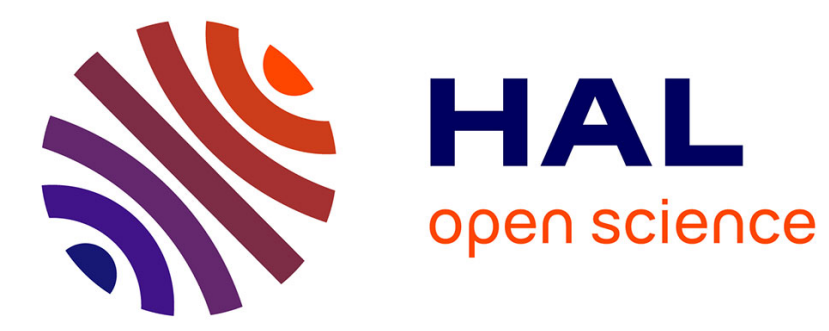

\title{
Adapted Output Space-Mapping Technique for a Bi-Objective Optimization
}

Tuan-Vu Tran, F. Moussouni, Stephane Brisset, P. Brochet

\section{To cite this version:}

Tuan-Vu Tran, F. Moussouni, Stephane Brisset, P. Brochet. Adapted Output Space-Mapping Technique for a Bi-Objective Optimization. IEEE Transactions on Magnetics, 2010, 46 (8), pp.2990 - 2993. 10.1109/TMAG.2010.2043343 . hal-01929429

\section{HAL Id: hal-01929429 \\ https://hal.science/hal-01929429}

Submitted on 21 Nov 2018

HAL is a multi-disciplinary open access archive for the deposit and dissemination of scientific research documents, whether they are published or not. The documents may come from teaching and research institutions in France or abroad, or from public or private research centers.
L'archive ouverte pluridisciplinaire $\mathbf{H A L}$, est destinée au dépôt et à la diffusion de documents scientifiques de niveau recherche, publiés ou non, émanant des établissements d'enseignement et de recherche français ou étrangers, des laboratoires publics ou privés. 


\title{
Adapted Output Space-Mapping Technique for a Bi-Objective Optimization
}

\author{
T. V. Tran, F. Moussouni, S. Brisset, and P. Brochet \\ Univ. Lille Nord de France, F-59000 Lille, France \\ ECLille, L2EP, F-59650, Villeneuve d'Ascq, France
}

\begin{abstract}
Multi-objective optimizations by means of 3D finite element models result in very high computation burden. To have an affordable computation cost, the output space-mapping technique is applied with a new method where the scalar correction of the model outputs is replaced by a set of corrective functions. This method is used for the bi-objective optimization of a transformer and allows finding the complete Pareto optimal set in less than two days on a laptop.
\end{abstract}

Index Terms - Space-mapping, Multi-objective optimization, 3D finite element model, Transformer.

\section{INTRODUCTION}

$\mathrm{T}$ HE OPTIMAL design of electromagnetic devices is a complex and complicated task. A way to formulate the problem is to find the trade-off between conflicting goals. Solving this problem requires building the Pareto optimal set with accuracy. With two objectives, the Pareto optimal set can be easily drawn and helps the designer to find a good solution. Many methods are able to find the solutions of a bi-objective optimization: scalar methods such as the well-known weighted sum (WS), $\varepsilon$-constraint methods, etc. [1]; and stochastic methods such as non-dominated sorting genetic algorithm II (NSGA-II), niched Pareto genetic algorithm (NPGA), etc. [2]. Unfortunately, those methods require a very high number of model evaluations. This is not compatible with the use of a 3D FE magneto-thermal model that requires 2 hours [3].

The purpose of space-mapping (SM) [4]-[7] is to align a coarse model and a fine model to reduce the computation time. In electromagnetic, the fine model is a $2 \mathrm{D}$ or $3 \mathrm{D} \mathrm{FEM}$ and the coarse model is often a lumped mass or analytical model. The Manifold-Mapping [6] and Output Space-Mapping (OSM) [7] techniques are the most recent and effective methods. Unfortunately, no SM algorithm has been developed or adapted to multi-objective optimization problem. The aim of this paper is to adapt the OSM to bi-objective optimization problems.

First, the bi-objective optimization of a safety transformer [3] is presented and the designer's dilemma is raised. Section III proposes a new method to solve this problem that is applied in section IV to the bi-objective optimization of the safety transformer. Finally, some concluding remarks are given.

\section{OPTIMIZATION PROBLEM}

The safety isolating transformer is a one-phase step-down transformer. It uses grain-oriented E-I laminations. The primary and secondary windings are both wound around the frame surrounding the central core (Fig.1).

It has been selected as a simple practical example capable to

Manuscript received December 23, 2009. Corresponding author: S. Brisset (e-mail: stephane.brisset@ec-lille.fr).

Digital Object Identifier inserted by IEEE provide a quantitative evaluation of the savings possible with the proposed procedure.

The bi-objective optimization problem of a safety isolating transformer contains 7 design variables. There are three parameters $a, b, c$ for the shape of the lamination, one for the frame $d$, two for the section of conductors $S_{1}, S_{2}$, and one for the number of primary turns $n_{l}$. The geometrical parameters and section of conductors are shown in Fig. 1.

The bi-objective optimization problem is expressed as:

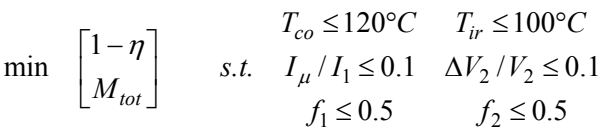

$$
\begin{aligned}
& 3 \mathrm{~mm} \leq a \leq 30 \mathrm{~mm} \quad 14 \mathrm{~mm} \leq b \leq 95 \mathrm{~mm} \\
& \text { with } \quad 6 \mathrm{~mm} \leq c \leq 40 \mathrm{~mm} \quad 10 \mathrm{~mm} \leq d \leq 80 \mathrm{~mm} \\
& 200 \leq n_{1} \leq 1200 \quad 0.15 \mathrm{~mm}^{2} \leq S_{1,2} \leq 19 \mathrm{~mm}^{2}
\end{aligned}
$$

There are 6 non-linear inequality constraints in this problem. The copper and iron temperatures $T_{c o}, T_{i r}$ should be less than $120^{\circ} \mathrm{C}$ and $100^{\circ} \mathrm{C}$, respectively. The magnetizing current $I_{\mu} / I_{1}$ and drop voltage $\Delta V_{2} / V_{2}$ should both be less than $10 \%$. Finally, the filling factors of both coils $f_{1}$ and $f_{2}$ should both be lower than 0.5 . The objective functions are to maximize the efficiency $\eta$ and to minimize the total mass $M_{t o t}$ of iron and copper materials. All electric and thermal quantities are computed at full-load.

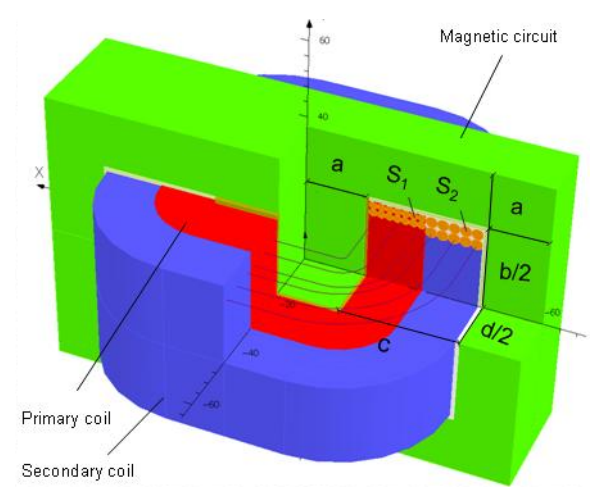

Fig.1. Bi-objective optimization problem of a safety isolating transformer 


\section{A. Coarse and fine models}

The multi-physical phenomena within the transformer are electric, magnetic and thermal. They are modeled by a lumped-mass (coarse) model (LM) and a 3D finite element (fine) model (3D FE) [3].

The LM model is built with the assumption that the voltage drop due to the magnetizing current is neglected. Therefore the maximal induction depends on the primary voltage. The computational time of the coarse model is very short $(50 \mathrm{~ms}$ on an Intel Pentium M 2.13 GHz laptop).

The 3D FE magneto-thermal model is built with the assumption that all magnetic and electric quantities are sinusoidal. The iron loss is computed with Steinmetz formula described in [3] and the leakage inductances are calculated with the magnetic energy. Full-load and no-load simulations are used to compute all characteristics of the safety isolating transformer. The 3D FE model with magneto-thermal coupling requires a very expensive computational time (about 2 hours on an Intel Pentium M $2.13 \mathrm{GHz}$ laptop).

\section{B. Designer's dilemma}

Generating 100 optimal solutions of the Pareto optimal set by means of the 3D FE model requires 10000 hours while 5 minutes only are needed with the LM model. In fact, as solving 3D FE model is very expensive only six points are computed and considered as a reference set (Fig. 2). The points are found by using the mono-objective OSM technique to reduce the computation time. The LM model is used to build an extended Pareto optimal set in a short time. It is obvious that this last optimal set is far from the reference set.

A first solution to this dilemma is to interpolate the reference set. Unfortunately, no information on the design parameters can be obtained because the interpolation can only be made in the objective space.

Therefore, the authors propose to adapt OSM technique for bi-objective optimization problems in order to generate an extended and accurate Pareto optimal set in less than 100 hours.

\section{AdAPTED SPACE-MAPPING TEChNIQUE}

\section{A. Bi-objective optimization algorithm}

According to the state of the art, the $\varepsilon$-constraint method is a useful approach to build a Pareto optimal set [1]. Moreover, this method may reach Pareto optimal solutions in the nonconvex region. The $\varepsilon$-constraint method consists to transform the multi-objective problem in a single-objective problem. Among the objectives, one is kept and the others are transformed in inequality constraints:

$$
x^{*}=\min _{x \in X} f_{i}(x) \quad \text { s.t. } \quad f_{j \neq i}(x) \leq \varepsilon_{j} \quad \text { and } \quad k_{f}(x) \leq 0
$$

where $f_{i}$ is the objective kept, $f_{j \neq i}$ are the other objectives, $k_{f}$ are the ordinary constraints, and $\varepsilon_{j}$ are the threshold values. By varying $\varepsilon_{j}$ between $\varepsilon_{j}^{\min }$ and $\varepsilon_{j}^{\max }$, the whole Pareto optimal set may be found. Two mono-objective

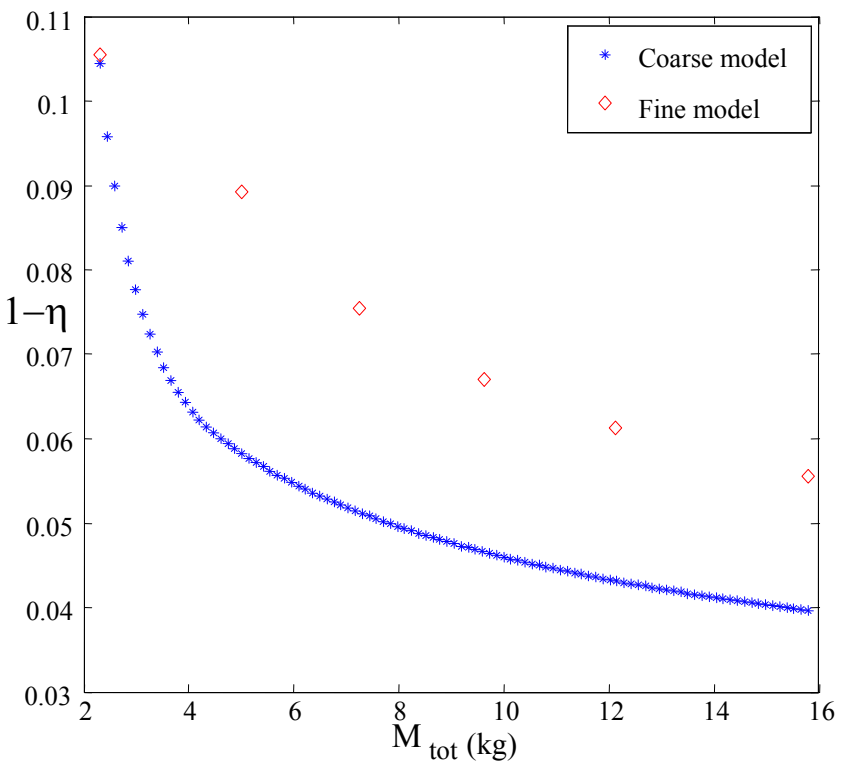

Fig.2. Pareto optimal set using the coarse and fine models

optimizations are computed to determine the upper and lower threshold values. $\varepsilon_{j}^{\min }$ is found by minimizing $f_{j \neq i}$ and $\varepsilon_{j}^{\max }$ is found by minimizing $f_{i}$. Using the $\varepsilon$-constraint method with the coarse model, a Pareto front with 100 points is quickly built and shown in Fig. 2 .

\section{B. Space-mapping techniques}

Space mapping techniques aim to use both coarse and fine models to reduce the computational time and increase the accuracy of the obtained solution [4]-[7].

In general, the coarse computationally cheaper model is denoted by $c(z) \in R^{m}$ with $z \in Z \subset R^{n}$, and the fine computationally expensive model is denoted by $f(x) \in R^{m}$ with $x \in X \subset R^{n}$. In practical engineering, the coarse and fine model spaces are often the same, i.e. $z \equiv x \in X \subset R^{n}$. The non-linear constraints computed by the coarse and the fine models are $k_{c}(z)$ and $k_{f}(x)$, respectively. The set of variables $x^{*}$ represents the solution of a given optimization problem:

$$
x^{*}=\underset{x \in X}{\arg \min }\|f(x)-y\| \quad \text { s.t. } \quad k_{f}(x) \leq 0
$$

where $y \in R^{m}$ denotes a vector of design specifications and can be zeros in the case of a minimization. In practice, solving (3) is very expensive. Therefore, the faster optimization problem based on the coarse model is preferred:

$$
z^{*}=\underset{z \in Z}{\arg \min }\|c(z)-y\| \quad \text { s.t. } \quad k_{c}(z) \leq 0
$$

One approach of SM techniques, called Output SpaceMapping (OSM) [7] consists to modify the coarse model by 
adding some corrective coefficients $\theta \in \Theta \subset R^{p}, p$ is the number of responses (objectives and constraints) computed by the fine model, in order to align the results of the coarse model with those of the fine model. The coefficients are updated at each iteration to minimize the discrepancy between both models:

$$
\theta^{(j+1)}=\underset{\theta \in \Theta}{\arg \min }\left\|\left[\begin{array}{c}
c\left(x^{(j)}, \theta\right) \\
k_{c}\left(x^{(j)}, \theta\right)
\end{array}\right]-\left[\begin{array}{c}
f\left(x^{(j)}\right) \\
k_{f}\left(x^{(j)}\right)
\end{array}\right]\right\|
$$

Then, they are introduced in the coarse model to compute a new solution $x^{(j+1)}$ for the next iteration:

$$
x^{(j+1)}=\underset{x \in X}{\arg \min }\left\|c\left(x, \theta^{(j+1)}\right)-y\right\| \quad \text { s.t. } k_{c}\left(x, \theta^{(j+1)}\right) \leq 0
$$

After that, a simulation with $x^{(j+1)}$ by the fine model is performed to compute the fine responses $f\left(x^{(i+1)}\right)$ and $k_{f}\left(x^{(i+1)}\right)$. The algorithm stops when all the corrective coefficients are unchanged from one iteration to the next one.

\section{Corrective spline functions}

A corrective coefficient is introduced for each objective and constraint therefore the corrected coarse model has the following expression:

$$
\left[\begin{array}{c}
c(x, \theta) \\
k_{c}(x, \theta)
\end{array}\right]=\operatorname{Diag}(\theta(\varepsilon)) \cdot\left[\begin{array}{c}
c(x) \\
k_{c}(x)
\end{array}\right]
$$

In the case of a mono-objective optimization only a set of $p$ scalar coefficients is searched ( $p$ is the number of objectives and constraints computed with the fine model).

$$
\theta^{(j+1)}=\left[\begin{array}{c}
c\left(x^{(j)}\right) / f\left(x^{(j)}\right) \\
k_{c}\left(x^{(j)}\right) / k_{f}\left(x^{(j)}\right)
\end{array}\right]
$$

where the indices $f$ and $c$ denote the fine and coarse model respectively.

In the case of a multi-objective optimization, the $p$ corrective coefficients' values must be changed for each solution from the Pareto set, i.e. for each value of the threshold value $\varepsilon \in\left[\varepsilon_{\min }, \varepsilon_{\max }\right]$. So that these $p$ coefficients are replaced by $p$ corrective functions of $\varepsilon$ :

$$
\theta^{(j+1)}(\varepsilon)=\left[\begin{array}{c}
S_{i}(\varepsilon) \\
\vdots
\end{array}\right]
$$

where $S(\varepsilon)$ is a spline cubic interpolation function. The spline cubic functions avoid the oscillations that appear in the polynomial interpolation approach when the order is high.

Since the mapping functions are defined, they are used by the multi-objective algorithm to correct the coarse model and to obtain a new Pareto optimal set.

The following section presents the proposed algorithm that combines OSM and $\varepsilon$-constraint algorithm.

\section{D.Proposed algorithm}

At the beginning of the algorithm $(j=0)$, all the corrective coefficients are initialized to unity:

$$
\theta^{(0)}=I
$$

At each iteration, a multi-objective optimization is performed by using the $\varepsilon$-constraint method and the corrected coarse model in order to obtain a new Pareto optimal set in a short time:

$$
\begin{aligned}
& \min _{x \in X} c_{1}\left(x, \theta^{(j)}(\varepsilon)\right) \quad \text { s.t. } \begin{array}{l}
k_{c}\left(x, \theta^{(j)}(\varepsilon)\right) \leq 0 \\
c_{2}\left(x, \theta^{(j)}(\varepsilon)\right) \leq \varepsilon
\end{array} \\
& \text { and } \varepsilon_{\min } \leq \varepsilon \leq \varepsilon_{\max } \quad \text { takes } 30 \text { values }
\end{aligned}
$$

Then, $\left(2^{j}+1\right)$ solutions $x^{*}$ belonging to the Pareto optimal set are chosen in order to compute the responses of the fine model $f\left(x_{i}^{*}\right)$ and $k_{f}\left(x_{i}^{*}\right)$. Note that only $2^{j-1}$ points are new. To establish the corrective functions at the next iteration, the new points are at the center of the intervals, in the same way as the Dichotomy method.

If the following condition is checked, the algorithm stops:

$$
\left\|\left[\begin{array}{c}
c\left(x^{*}, \theta^{(j+1)}(\varepsilon)\right) \\
k_{c}\left(x^{*}, \theta^{(j+1)}(\varepsilon)\right)
\end{array}\right]-\left[\begin{array}{c}
c\left(x^{*}, \theta^{(j)}(\varepsilon)\right) \\
k_{c}\left(x^{*}, \theta^{(j)}(\varepsilon)\right)
\end{array}\right]\right\| \leq \tau
$$

where $\tau$ is the required accuracy. If the corrected coarse model responses are not close enough to the fine model ones, the algorithm continues by updating the corrective functions (8) with the new points.

To summarize, the algorithm carries out the following main steps:

0. initialization: $j=0, \theta^{(0)}=I$

1. build a Pareto optimal set by using the $\varepsilon$ constraints method and the corrected coarse model $c\left(x, \theta^{(j)}\right)$ to solve (11)

2. choose $2^{j}+1$ points on the Pareto optimal set.

3. evaluate the fine model with the chosen points to compute $f(x)$ and $k_{f}(x)$

4. update the $p$ corrective functions $\theta^{(j+1)}(\varepsilon)$.

5. if (12) stop the algorithm else $j=j+1$, go to 1 .

\section{APPLICATION CASE}

The bi-objective optimization problem of the safety isolating transformer presented in section II is solved with the proposed algorithm.

For this optimization problem, two constraints (filling factors $f_{1}, f_{2}$ ) among six and one objective (total mass $M_{t o t}$ ) among two are not evaluated by the 3D FE model. Indeed, both filling factors and the total mass are analytically computed. So that, five responses $\eta, T_{c o}, T_{i r}, I_{\mu} / I_{1}$, and $\Delta V_{2} / V_{2}$ need 3D FE computation, i.e. only five values of corrective coefficients $\left[\theta_{1}, \theta_{2}, \theta_{3}, \theta_{4}, \theta_{5}\right]$ are computed for each threshold value $\varepsilon \in\left[\varepsilon_{\min }, \varepsilon_{\max }\right]$.

The losses 1- $\eta$ are $c_{1}$ in (11) and the total mass $M_{t o t}$ is $c_{2}$. Therefore, the bi-objective optimization problem (1) becomes a set of 30 mono-objective optimization problems with one 
additional constraint on the total mass $M_{t o t}$ :

$$
\begin{array}{ll}
\min & 1-\eta \\
& T_{c o} \leq 120^{\circ} \mathrm{C} \quad T_{i r} \leq 100^{\circ} \mathrm{C} \\
\text { s.t. } & I_{\mu} / I_{1} \leq 0.1 \quad \Delta V_{2} / V_{2} \leq 0.1 \quad \text { and } \quad M_{\text {tot }} \leq \varepsilon \\
& f_{1} \leq 0.5 \quad f_{2} \leq 0.5 \\
& 3 \mathrm{~mm} \leq a \leq 30 \mathrm{~mm} \quad 14 \mathrm{~mm} \leq b \leq 95 \mathrm{~mm} \\
\text { with } & 6 \mathrm{~mm} \leq c \leq 40 \mathrm{~mm} \quad 10 \mathrm{~mm}^{2} \leq d \leq 80 \mathrm{~mm} \\
& 200 \leq n_{1} \leq 1200 \quad 0.15 \mathrm{~mm}^{2} \leq S_{1,2} \leq 19 \mathrm{~mm}^{2}
\end{array}
$$

At initialization $(j=0)$, the coarse model is not corrected yet and the corrective functions are all equal to one. Two points at $\varepsilon_{j}^{\min }$ and $\varepsilon_{j}^{\max }$ are computed with the fine model to test the stop criteria and build the corrective functions for the next iteration. At iteration 1, the corrective functions are now linear (Fig. 4) and a new optimal set is obtained. In Fig. 4, the corrective function is given only for the efficiency of the transformer, i.e. $\theta_{1}$.

At each iteration, one new point is added at the middle of each couple of points, resulting in $2^{j-1}$ new points amongst a total of $\left(2^{j}+1\right)$ points, i.e. 3D FE model evaluations.

Fig. 3 shows the Pareto optimal set obtained by using the corrective functions. 30 points are given at each iteration.

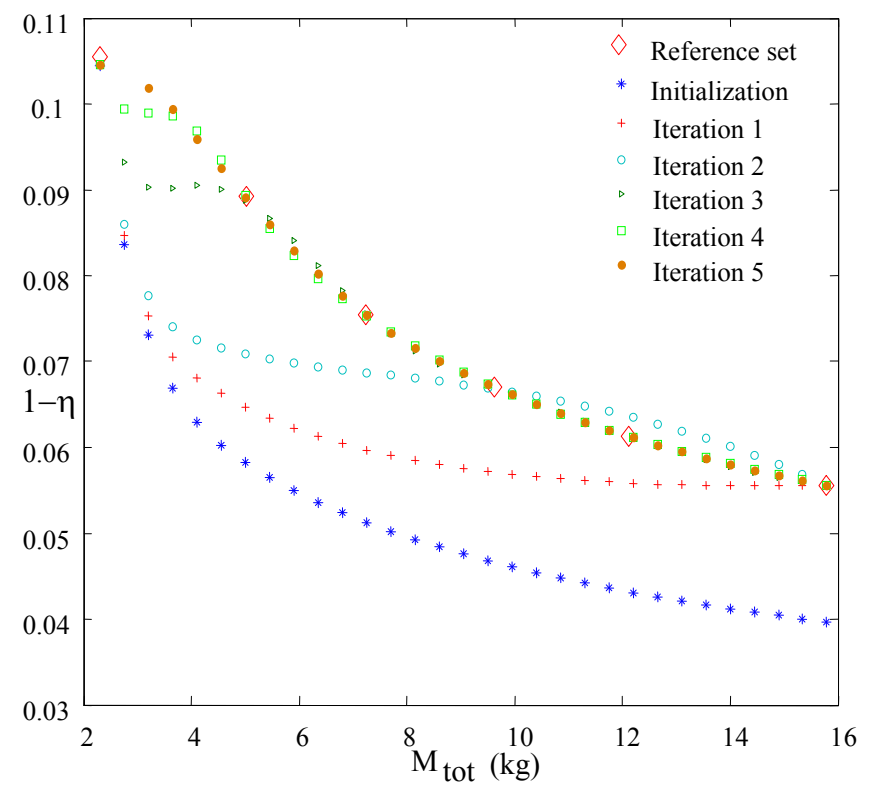

Fig.3. Pareto optimal set obtained at each iteration

At the end of the optimization, a Pareto optimal set very close to the reference set is found.

Finally, only 17 3D FE simulations are needed to obtain an accurate Pareto optimal set. The computational time of the proposed algorithm is approximately 34 hours (1.4 day) on an Intel Pentium M $2.13 \mathrm{GHz}$ laptop. The reference Pareto optimal set requires 60 hours (2.5 days) on the same machine. To have an accurate Pareto optimal set by using the 3D FE model only, approximately 10,000 hours (14 months) are required. All the computed points are shown with circles in

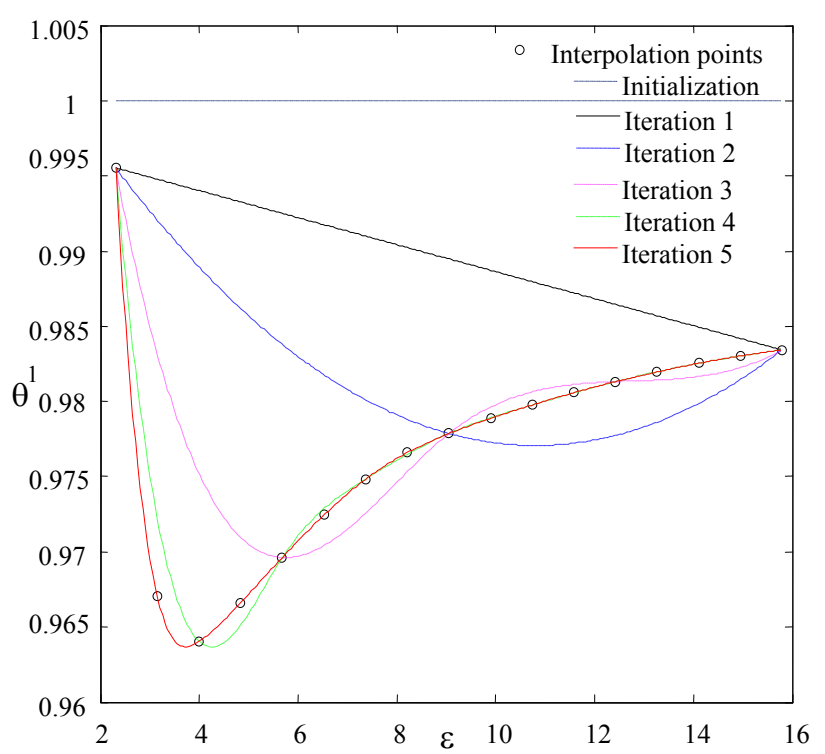

Fig.4. Corrective functions for the efficiency at each iteration.

Fig. 4.

\section{V.CONCLUSION}

The output space-mapping technique is adapted to provide a practical way to build an accurate Pareto optimal set in biobjective optimization using full 3D coupled finite element model and keeping the computational time in an acceptable limit. In addition to the 3D FE model, a simpler one provided with corrective coefficients has been used. Cubic spline interpolation functions are used to predict the values of the corrective coefficients. By using these corrective functions, the $\varepsilon$-constraints bi-objective optimization algorithm gives an extended and accurate Pareto optimal set in less than 2 days.

\section{REFERENCES}

[1] A. Murano, A. Passaro, N. M. Abe, A. J. Preto, S. Stephany, "Multiobjective Optimization of Electro-optic Modulators by Using the $\varepsilon$-constraint Method", proceedings of CEFC 2008, May, 2008, Athens, Greece.

[2] A. H. F. Dias, and J. A. de Vasconcelos, "Multiobjective Genetic Algorithms Applied to Solve Optimization Problems", IEEE Transactions on Magnetics, vol. 38, no. 2, pp. 1133-1136, 2002.

[3] T. V. Tran, S. Brisset and P. Brochet, "A Benchmark for MultiObjective, Multi-Level and Combinatorial Optimizations of a Safety Isolating Transformer", proceedings of COMPUMAG 2007, June 2007, Aachen, Germany, all materials can be downloaded at http://12ep.univlille1.fr/come/benchmark-transformer.htm.

[4] H. Choi, D. Kim, I. Park, and S. Hahn, "A New Design Technique of Magnetic Systems using Space Mapping Algorithm”, IEEE Trans. on Magn., vol. 37, no 5, pp. 3627-3630, 2001.

[5] J. W. Bandler, Q. S. Cheng, S. A. Dakroury, A. S. Mohamed, M. H. Bakr, K. Madsen, and J. Sondergaard, "Space Mapping: The State of the Art”, IEEE Trans. Microwave Theory Tech., vol. 52, no. 1, pp. 337-361, 2004.

[6] D. Echeverria, D. Lahaye, L. Encica, E. A. Lomonova, P. W. Hemker, and A. J. A. Vandenput, "Manifold-Mapping Optimization Applied to Linear Actuator Design”, IEEE Trans. on Magn., vol. 42, no. 4, pp. 1183-1186, 2006.

[7] L. Encica, J. J. H. Paulides, E. A. Lomonova, A. J. A. Vandenput, "Aggressive Output Space-Mapping Optimization for Electromagnetic Actuators", IEEE Transactions on Magnetics, vol. 44, no. 6, pp. 11061109, 2008. 